\title{
Coupling of Carbon Dioxide with Neat Propylene Oxide Catalyzed by Aminebisphenolato Cobalt(II)/(III) Complexes and Ionic Co-catalysts
}

This is a postprint version of this article. Please cite as follows:

Lisa N. Saunders, Nduka Ikpo, Chad F. Petten, Uttam Kumar Das, Louise N. Dawe, Christopher M. Kozak, Francesca M. Kerton, Coupling of carbon dioxide with neat propylene oxide catalyzed by aminebisphenolato cobalt(II)/(III) complexes and ionic co-catalysts, Catalysis Communications, Volume 18, 10 February 2012. http://dx.doi.org/10.1016/j.catcom.2011.11.036.

Lisa N. Saunders, ${ }^{a}$ Nduka Ikpo, ${ }^{a}$ Chad F. Petten, ${ }^{a}$ Uttam Kumar Das, ${ }^{a}$ Louise N. Dawe, ${ }^{b}$ Christopher M. Kozak ${ }^{a}$ and Francesca M. Kerton ${ }^{a}$ *

a. Centre for Green Chemistry and Catalysis, Department of Chemistry, Memorial University, St. John's, NL, A1B 3X7, Canada

b. X-ray Crystallography Laboratory, Centre for Chemical Analysis, Research and Training, and Department of Chemistry, Memorial University, St. John's, NL, A1B 3X7, Canada

*Corresponding authors. Email addresses: ckozak@mun.ca, fkerton@mun.ca

Keywords: Epoxide, Carbon Dioxide, Cobalt, Cyclic carbonate, N,O-ligand

\section{Highlights}

- Oxidation state of cobalt center plays crucial role in determining activity

- Activity is greater for complexes of pyridyl-containing ligand

- Ionic co-catalysts are more effective than neutral Lewis bases with these catalysts

- High TON achieved at room temperature under solvent-free condition

\begin{abstract}
Cobalt complexes of tetradentate amine-phenolate ligands were studied for their potential in coupling carbon dioxide with propylene oxide under neat reaction conditions. Cobalt(II) complexes afforded catalytic systems with higher TONs than analogous cobalt(III) compounds. Tetrabutylammonium bromide (TBAB) and $\mathrm{PPN}^{+} \mathrm{N}_{3}{ }^{-}$were effective co-catalysts whereas N,N-dimethylaminopyridine (DMAP) shut down reactivity. Ligands containing a pendant pyridyl donor afforded more active catalysts than those containing dimethylamine
\end{abstract}


groups. Reactions proceeded well at room temperature under moderate pressures of carbon dioxide. 


\section{Graphical Abstract}

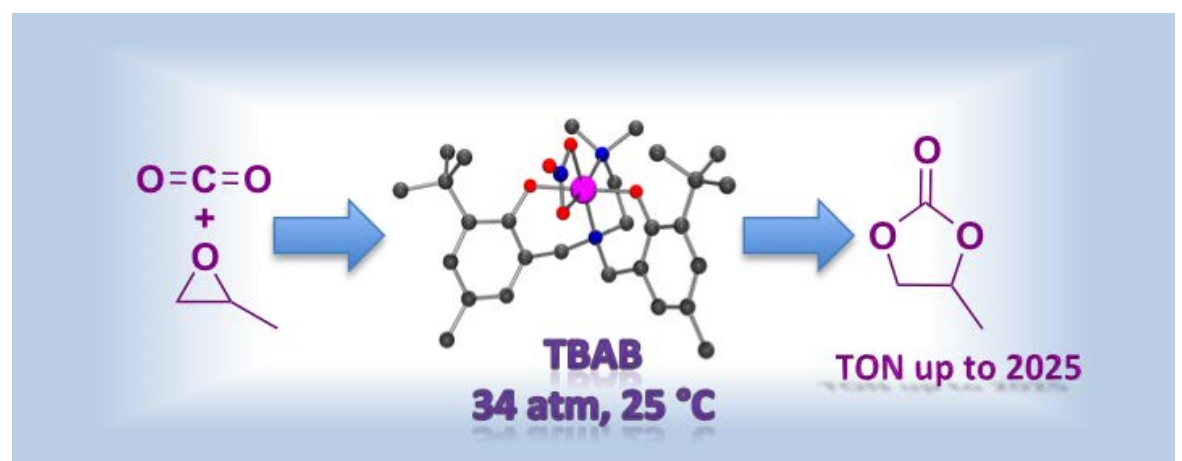

\section{Introduction}

Carbon dioxide is an abundant and cheap C1 feedstock and there is continued interest in its chemical fixation, particularly its reactions with epoxides to form polycarbonates or cyclic carbonates [1-5]. The cyclic carbonates can be used as polar aprotic solvents and intermediates in organic synthesis. Cobalt(III) complexes of salen (N,N'-bis(3,5-di-tert-butylsalicylidene)-1,2-cyclohexanediamine) ligands have been particularly effective in the alternating copolymerization of propylene oxide (PO) and carbon dioxide [6, 7]. Darensbourg and Moncada recently reported that a related cobalt(II) complex in the presence of an anionic initiator could catalyze oxetane-carbon dioxide copolymerization [8]. Also, Williams and co-workers have developed remarkable dicobalt (II/II and II/III) and tricobalt (II/II/II) complexes of an amido-phenolato macrocyclic ligand for cyclohexene oxide-carbon dioxide copolymerization under 1 atm carbon dioxide [9]. In contrast to these examples, cobalt(II) salen complexes containing a binapthyl backbone in the presence of neutral Lewis base co-catalysts perform cycloaddition reactions of carbon dioxide with epoxides to yield cyclic carbonates with TON 800-913 [10]. Subsequently, it was shown that in these examples the phenolic ligands themselves could perform such reactions and similar TONs could be obtained without the need for a metal [11]. A range of bifunctional cobalt-salen complexes have been reported that under the appropriate conditions either copolymerize or form cyclic carbonates from propylene oxide and carbon dioxide [12-15]. We have recently reported a series of well-characterized Co(II) complexes 
[16]. Given the precedent for carbon dioxide fixation using cobalt complexes of N,O-ligands, herein, we report our investigation of amine-phenolato Co(II) and Co(III) complexes, Fig. 1, for the coupling reaction of propylene oxide and carbon dioxide at room temperature under solvent-free conditions.

\section{Experimental}

\subsection{Materials}

Reagents were purchased from Alfa Aesar or Sigma Aldrich and all except propylene oxide (PO) were used without further purification. PO was distilled from $\mathrm{CaH}_{2}$. Dichloromethane was purified using an MBraun solvent purification system. Supercritical fluid grade carbon dioxide was acquired from Air Liquide.

\subsection{Synthesis and characterization of cobalt complexes.}

Cobalt(II) complexes, including 3, were prepared according to literature procedures [16]. Co(III) complexes were prepared via oxidation using $\mathrm{AgNO}_{3}$ or $\mathrm{AgO}_{3} \mathrm{SCF}_{3}$. As an example, details for the preparation of $\mathrm{Co}^{\mathrm{III}}\left[\mathrm{O}_{2} \mathrm{NN}^{\prime}\right]^{\mathrm{BuNMe}}\left(\mathrm{NO}_{3}\right.$ ) (Fig. 1. Compound 7) follows.

$\mathrm{Co}^{\mathrm{II}}\left[\mathrm{O}_{2} \mathrm{NN}^{\prime}\right]^{\mathrm{BuNMe} 2}, \mathbf{1}(0.52 \mathrm{~g}, 0.88 \mathrm{mmol})$, was added to a $50 \mathrm{~mL}$ round-bottomed flask. Dry dicholoromethane $(25 \mathrm{~mL})$ was added to dissolve the complex and a brown solution formed. Silver nitrate $(0.20 \mathrm{~g}, 0.96 \mathrm{mmol})$ was added, the flask was wrapped with aluminum foil and the mixture was stirred open to air at room temperature for $8 \mathrm{~h}$. The resulting dark brown mixture was filtered through a glass frit to remove precipitated silver, and the solvent was removed under vacuum. The product, $\mathrm{Co}^{\mathrm{II}}\left[\mathrm{O}_{2} \mathrm{NN}^{\prime}\right]^{\mathrm{BuNMe}} 2\left(\mathrm{NO}_{3}\right) 7$, was isolated as a dark brown powder (yield $0.51 \mathrm{~g}, 0.78 \mathrm{mmol}, 89 \%$ ). Crystals of 7 suitable for single-crystal X-ray diffraction analysis were grown from a saturated solution in acetone stored at $-35^{\circ} \mathrm{C}$.

Anal. Calcd for $\mathrm{C}_{34} \mathrm{H}_{54} \mathrm{CoN}_{3} \mathrm{O}_{5} ; \mathrm{C}, 63.44 ; \mathrm{H}, 8.46 ; \mathrm{N}, 6.53$. Found: $\mathrm{C}, 63.02 ; \mathrm{H}, 8.53 ; \mathrm{N}, 6.62 .{ }^{1} \mathrm{H}$ NMR ( $\left.\mathrm{CDCl}_{3}, 500 \mathrm{MHz}\right): \delta 1.26(\mathrm{~s}, 18 \mathrm{H}), 1.45(\mathrm{~s}, 18 \mathrm{H}), 2.75(\mathrm{br} \mathrm{s}, 2 \mathrm{H}), 2.80(\mathrm{~s}, 6 \mathrm{H}), 2.89(\mathrm{br}$, $2 \mathrm{H}), 2.99\left(\mathrm{~d},{ }^{2} J=13.8 \mathrm{~Hz}, 2 \mathrm{H}\right), 3.52\left(\mathrm{~d},{ }^{2} J=13.7 \mathrm{~Hz}, 2 \mathrm{H}\right), 6.90(\mathrm{~s}, 2 \mathrm{H}), 7.20(\mathrm{~s}, 2 \mathrm{H}) .{ }^{13} \mathrm{C}$ NMR 
( $\left.\mathrm{CDCl}_{3}, 125 \mathrm{MHz}\right): 29.8,29.9,34.2,34.9,45.2,50.8,63.0,64.8,118.9,121.9,123.7,136.3$, 141.8, 156.2. MS (ESI): $m / z(\%) 644\left(65, \mathrm{M}^{+}\right)$.

\subsection{General procedure for coupling reaction of $\mathrm{PO}$ and $\mathrm{CO}_{2}$}

All reactions were performed in a $300 \mathrm{~mL}$ stainless steel pressure vessel (Parr) equipped with an overhead stirrer. The pressure vessel was heated under vacuum overnight to remove trace water. Under a nitrogen atmosphere, cobalt complex (0.05 $\mathrm{mmol})$, PO (7.5 mL, $107 \mathrm{mmol})$ and co-catalyst $(0.05 \mathrm{mmol})$ were mixed in a vial and then the contents of the vial transferred to the pressure vessel. The reactor was pressurized with $\mathrm{CO}_{2}$ to $34 \mathrm{~atm}$ and stirred. In some cases, a moderate increase in temperature (reaction exotherm) was noted. After $12 \mathrm{~h}$, the reactor was cooled in an ice bath and vented. An aliquot of the reactor contents was taken for analysis via ${ }^{1} \mathrm{H}$ NMR. Propylene carbonate could be isolated from the reaction mixture via filtration through a short plug of silica gel, to remove the catalysts, and concentration under vacuum.

\section{Results and discussion}

\subsection{Effect of ligand and co-catalyst on cycloaddition of $\mathrm{PO}$ and $\mathrm{CO}_{2}$}

Initial studies were performed using the neutral Co(II) complexes 1-4 (Fig. 1) as catalysts with a range of co-catalysts. As tetrabutylammonium halides have been reported as efficient catalysts and solvents for the reaction concerned [17], a control reaction using TBAB alone was also performed (Table 1, entry 1 ) for comparison with reactions in the presence of cobalt amine-phenolato complexes. It should also be noted that PPN salts alone have also been reported to yield cyclic carbonates under neat reaction conditions at high temperature [18]. As TBAB afforded a slightly superior catalyst in this study, we did not perform a control reaction for PPN azide alone. Compounds $\mathbf{1}$ and $\mathbf{3}$ contain a pyridyl group as the pendant donor, whereas $\mathbf{2}$ and $\mathbf{4}$ contain a dialkylamine group. The nature of this pyridyl donor group is important for achieving high TON in the cycloaddition reaction (Table 1, entries 2-5). This effect could be due to either electronic or steric effects as the pyridyl group is a stronger sigma-donor and less sterically demanding compared with the dimethylamine pendant group. Future research efforts will aim at understanding this 
difference through use of both experimental (e.g. X-ray photoelectron spectrsocopy) and computational methods. The alkyl substituents on the phenolate group also affect the catalytic activity observed with the $t$-amyl containing compounds showing greater activity compared with the $t$-butyl analogues.

Ionic co-catalysts were found to be essential in the reactions studied. A cobalt(II) compound alone did not convert the propylene oxide (Table 1, entry 6). PPN azide afforded a TON similar to that obtained with TBAB but use of 4-dimethylaminopyridine (DMAP), a widely used neutral Lewis base co-catalyst, did not afford an active catalytic system (Table 1, entries 7 and 8).

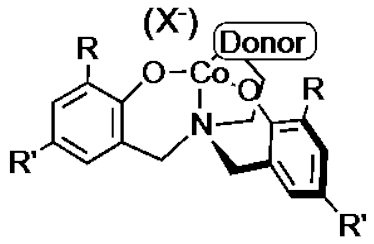

$$
\begin{aligned}
\text { 1, R } & =\mathrm{R}^{\prime}=t-\mathrm{Bu}, \text { Donor }=\mathrm{C}_{5} \mathrm{H}_{4} \mathrm{~N} \\
\mathbf{2}, \mathrm{R} & =\mathrm{R}^{\prime}=t-\mathrm{Bu}, \text { Donor }=\mathrm{NMe}_{2} \\
3, \mathrm{R} & =\mathrm{R}^{\prime}=t-\mathrm{Am}, \text { Donor }=\mathrm{C}_{5} \mathrm{H}_{4} \mathrm{~N} \\
4, \mathrm{R} & =\mathrm{R}^{\prime}=t-\mathrm{Am}, \text { Donor }=\mathrm{NEt}_{2} \\
\mathrm{R}^{\prime} & \\
5, \mathrm{R} & =\mathrm{R}^{\prime}=t-\mathrm{Bu}, \text { Donor }=\mathrm{C}_{5} \mathrm{H}_{4} \mathrm{~N}, \mathrm{X}^{-}=\mathrm{CF}_{3} \mathrm{SO}_{3}^{-} \\
6, \mathrm{R} & =\mathrm{R}^{\prime}=t-\mathrm{Bu}, \text { Donor }=\mathrm{C}_{5} \mathrm{H}_{4} \mathrm{~N}, \mathrm{X}^{-}=\mathrm{NO}_{3}^{-} \\
7, \mathrm{R} & =\mathrm{R}^{\prime}=t-\mathrm{Bu}, \text { Donor }=\mathrm{NMe}_{2}, \mathrm{X}^{-}=\mathrm{NO}_{3}^{-}
\end{aligned}
$$

Fig. 1. Schematic structure of Co(II) 1-4 and Co(III) 5-7 complexes used as catalysts in this study

\section{Table 1}

Reactivity of 1-7 towards carbon dioxide/PO cycloadditions with different co-catalysts ${ }^{\text {a }}$.

\begin{tabular}{llll}
\hline Entry & Complex & Co-catalyst & TON $^{\mathrm{b}}$ \\
\hline 1 & - & TBAB & 17 \\
2 & $\mathbf{1}$ & TBAB & 950 \\
3 & $\mathbf{2}$ & TBAB & 250 \\
4 & $\mathbf{3}$ & TBAB & 2025 \\
5 & $\mathbf{4}$ & TBAB $^{-}$ & 300 \\
6 & $\mathbf{1}$ & - & 0 \\
7 & $\mathbf{1}$ & PPN $^{+} \mathrm{N}_{3}^{-}$ & 800 \\
8 & $\mathbf{1}$ & DMAP $^{-}$ & 0 \\
9 & $\mathbf{5}$ & PPN $^{+} \mathrm{N}_{3}^{-}$ & 30 \\
10 & $\mathbf{6}$ & PPN $^{+} \mathrm{N}_{3}^{-}$ & 15 \\
11 & $\mathbf{7}$ & - & 65 \\
12 & $\mathbf{7}$ & TBAB $^{-}$ & 400 \\
13 & $\mathbf{7}$ & PPN $^{+} \mathrm{N}_{3}^{-}$ & 200 \\
14 & $\mathbf{7}$ & DMAP $^{-}$ & 30 \\
\hline
\end{tabular}


a Reaction conditions: Cobalt complex $(0.05 \mathrm{mmol})$, co-catalyst $(0.05 \mathrm{mmol}), \mathrm{T}=25^{\circ} \mathrm{C}, P_{\mathrm{CO} 2}$ $=34 \mathrm{~atm}, 12 \mathrm{~h}$. Yields were determined using ${ }^{1} \mathrm{H}$ NMR spectroscopy and isolated yields were in agreement (entries 4, 7 and 12). ${ }^{\mathrm{b}}$ Moles of propylene carbonate produced per mole cobalt complex.

Several reports have recently shown that bimetallic complexes of salen-type ligands can ring-open epoxides to yield polyethers.[19-21] It should be noted that in the current studies, no evidence for homopolymerization of PO was obtained. We have previously shown that $\mathbf{2}$ forms a PO adduct and NMR studies of $\mathbf{7}$ in the presence of either equimolar or excess quantities of PO show no evidence of coordination (i.e. broadening of PO signals) or reaction (i.e. signals for polyether). Furthermore, in all reactions reported herein, no evidence of polypropylene carbonate formation was observed.

\subsection{Effect of cobalt oxidation state on coupling of $\mathrm{PO}$ and $\mathrm{CO}_{2}$}

In previous studies, many researchers have found that cobalt(III) complexes are highly effective in epoxide-carbon dioxide copolymerization reactions. Therefore, oxidation reactions were performed and compounds 5-7 were isolated. 7 was characterized by X-ray crystallography and contains cobalt in an octahedral coordination environment with the nitrate anion coordinated in a terminal, bidentate fashion (Fig. 2).

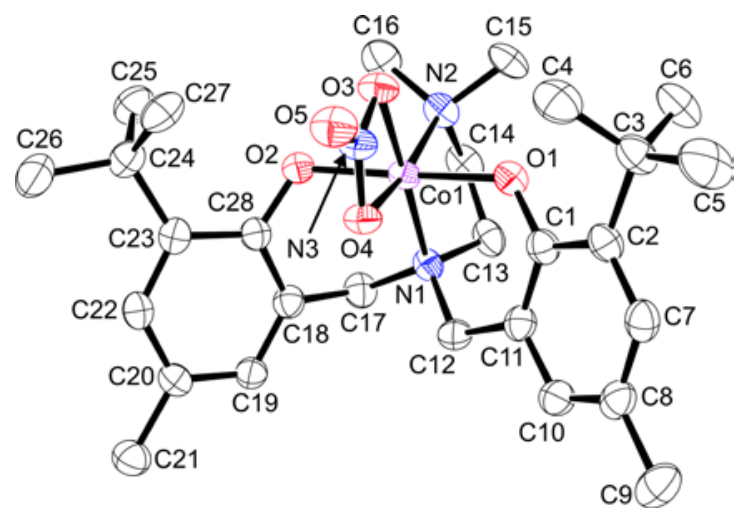

Fig. 2. Molecular structure of Co(III) complex (7) used as a catalyst in this study

Cycloaddition reactions using the cationic Co(III) complexes 5-7 were performed under identical conditions to their neutral Co(II) analogues. However, as compound $\mathbf{7}$ was 
structurally characterized by X-ray diffraction, this complex was studied in more detail than the other two. Compound $\mathbf{7}$ exhibits slight activity without the addition of a co-catalyst (Table 1, entry 11). Presumably, the nitrate anion is sufficiently nucleophilic to ring-open the epoxide substrate. In terms of co-catalysts, TBAB was once again superior compared with PPN azide and DMAP was found to inhibit the reactions (Table 1, entries 12-14). The Co(II) analogue of $\mathbf{7}$ is $\mathbf{1}$. Reactions using $\mathbf{1}$ with ionic co-catalysts have significantly higher TON than reactions using 7 . Therefore, oxidation state plays an important role in determining the activity of cobalt amine-phenolato complexes towards carbon dioxide fixation.

\section{Conclusions}

In summary, a new catalytic system for coupling of propylene oxide and carbon dioxide at room temperature is reported. The oxidation state of the cobalt center and the nature of the neutral pendant donor are of importance with regards to catalytic activity. The stronger-donating, planar pyridyl-donor group containing complexes were the most active studied and achieved high TONs (800-2025) with ionic co-catalysts. To the best of our knowledge, this is the first report of tripodal amine-phenolato cobalt complexes for coupling of an epoxide with carbon dioxide.

\section{Acknowledgments}

We are grateful to NSERC of Canada, Canada Foundation for Innovation, the Province of Newfoundland and Labrador (RDC NL) and Memorial University for financial support.

\section{Appendix A. Supplementary data}

Supplementary data (X-ray report) associated with this article can be found in the online version. CCDC 848223 contains the supplementary crystallographic data for complex 7. The data can be obtained free of charge on application to CCDC, 12 Union Road, Cambridge CB2 1EZ, UK (fax: +44 01223 336033; e-mail: deposit@ccdc.cam.ac.uk).

\section{References}

[1] M.R. Kember, A. Buchard, C.K. Williams, Chem. Commun. 47 (2011) 141-163. 
[2] D.J. Darensbourg, Chem. Rev. 107 (2007) 2388-2410.

[3] G.W. Coates, D.R. Moore, Angew. Chem., Int. Ed. 43 (2004) 6618-6639.

[4] M. North, R. Pasquale, C. Young, Green Chem. 12 (2010) 1514-1539.

[5] T. Sakakura, K. Kohno, Chem. Commun. (2009) 1312-1330.

[6] Z. Qin, C.M. Thomas, S. Lee, G.W. Coates, Angew. Chem., Int. Ed. 42 (2003) 5484-5487.

[7] C.T. Cohen, T. Chu, G.W. Coates, J. Am. Chem. Soc. 127 (2005) 10869-10878.

[8] D.J. Darensbourg, A.I. Moncada, Macromolecules 42 (2009) 4063-4070.

[9] M.R. Kember, A.J.P. White, C.K. Williams, Macromolecules 43 (2010) 2291-2298.

[10] Y.-M. Shen, W.-L. Duan, M. Shi, J. Org. Chem. 68 (2003) 1559-1562.

[11] Y.-M. Shen, W.-L. Duan, M. Shi, Eur. J. Org. Chem. (2004) 3080-3089.

[12] S.S.J.K. Min, J.E. Seong, S.J. Na, B.Y. Lee, Angew. Chem., Int.Ed. 47 (2008) 7306-7309.

[13] B. Liu, X. Zhao, H. Guo, Y. Gao, M. Yang, X. Wang, Polymer 50 (2009) 5071-5075.

[14] B. Liu, Y. Gao, X. Zhao, W. Yan, X. Wang, J. Polym. Sci., Part A: Polym. Chem. 48 (2010) 359-365.

[15] C.-X. Miao, J.-Q. Wang, Y. Wu, Y. Du, L.-N. He, ChemSusChem 1 (2008) 236-241.

[16] U.K. Das, J. Bobak, C. Fowler, S.E. Hann, C.F. Petten, L.N. Dawe, A. Decken, F.M. Kerton, C.M. Kozak, Dalton Trans. 39 (2010) 5462-5477.

[17] V. Calo, A. Nacci, A. Monopoli, A. Fanizzi, Org. Lett. 4 (2002) 2561-2563.

[18] W.N. Sit, S.M. Ng, K.Y. Kwong, C.P. Lau, J. Org. Chem. 70 (2005) 8583-8586.

[19] R.M. Thomas, P.C.B. Widger, S.M. Ahmed, R.C. Jeske, W. Hirahata, E.B. Lobkovsky, G.W. Coates, J. Am. Chem. Soc. 132 (2010) 16520-16525.

[20] P.C.B. Widger, S.M. Ahmed, W. Hirahata, R.M. Thomas, E.B. Lobkovsky, G.W. Coates, Chem. Commun. 46 (2010) 2935-2937.

[21] P.C.B. Widger, S.M. Ahmed, G.W. Coates, Macromolecules 44 (2011) 5666-5670. 